\title{
Automation of a water supply system for residences
}

\author{
Leandro da Mota Farias ${ }^{1}$, Rafael Souza da Silva²
}

${ }^{1,2}$ Centro Universitário do Norte (UNINORTE). Manaus - Amazonas - Brasil. Avenida Leonardo Malcher. No. 715.Centro. Manaus Amazonas. Fone: (92) 3212-5000.

E-mail: leandro_da_mota-sama@hotmail.com

Received: April 21'th, 2017

Accepted: May $28^{\text {th }}, 2017$

Published: June $30^{\text {th }}, 2017$

Copyright $(02016$ by authors and Institute of Technology Galileo of Amazon (ITEGAM). This work is licensed under the Creative Commons Attribution International License (CC BY 4.0).

http://creativecommons.org/lic enses/by/4.0/

\begin{abstract}
Water scarcity is increasingly haunting the world, and the outlook for the next few years is not enough for everyone to drink, thanks to the pollution and waste that grows every year for a population growing more and more on the globe. Water scarcity is not the only problem we face. In Brazil, the country with the largest freshwater reserve in the world, another major problem is the supply of water in the cities, in several regions the population faces an inefficient supply system, which presents faults in the water course the residence. The present work proposes an improvement in the residential water supply system that uses hydraulic pumps, automating the process of feeding the reservoirs of the residential, developing an autonomous system allowing the user to have control of the supply process in their property. The importance of the work is made by the difficulty encountered by residents of regions that suffer from the irregular water supply in their homes. The work was based on an experimental research, starting from a bibliographical research in several literatures as academic papers, researches and articles, focused on the study on water supply, hydraulic systems and automation. The studies were based on a residence in the northern part of Manaus/Amazon as, where there are irregularities in the water supply in this region, causing residents to use hydraulic systems to supply their residential reservoirs. In addition, a prototype for better observation of the automated operation of the small-scale supply process was developed for the study to perform better. Through an existing hydraulic system in the house, the automation process was carried out by the elaboration of an electrical system with the function of activating the pump through an Arduino ${ }^{\circledR}$ and for indicate the sensors responsible for the level of water in the reservoirs. A simple and practical monitoring system was developed based on an electrical principle and a hydraulic system. After the tests carried out, the model proposed in this work showed a simple and functional hydraulic supply system, facilitating for the user the monitoring of the water supply operation for the residence, the project was elaborated using elements already existing in the residence in order to reduce costs for the user, an autonomous system was implanted in the water supply process of the residence, thus enabling practicality in the activation of the hydraulic pump and the monitoring of levels in the reservoirs of the residence.
\end{abstract}

Keywords: Automation. Water Supply System. Hydraulic System.

\section{RESUMO}

A escassez de água assombra cada vez mais o mundo, e a previsão para os próximos anos não é de água potável suficiente para todos, graças a poluição e desperdício que cresce a cada ano por uma população que cresce cada vez mais no globo, mas a escassez de água não é o único problema que enfrentamos. No Brasil, o país com a maior reserva de água doce do mundo, outro grande problema presente é o de abastecimento nas cidades, em várias regiões a população enfrenta um sistema de abastecimento ineficiente, que apresenta falhas no curso da água, de sua captação até a residência. O presente trabalho propõe uma melhoria no sistema de abastecimento de água residencial que utilizam bombas hidráulicas, automatizando o processo de alimentação dos reservatórios das residenciais, desenvolvendo um sistema autônomo permitindo ao usuário ter o controle do processo de abastecimento em sua propriedade. A importância do trabalho se faz pela dificuldade encontrada por moradores de regiões que sofrem com o abastecimento irregular de água em suas casas. $\mathrm{O}$ trabalho foi elaborado com base em uma pesquisa experimental, partindo de uma pesquisa bibliográfica em diversas literaturas como trabalhos acadêmicos, pesquisas e artigos, voltados ao estudo sobre abastecimento de água, sistemas hidráulicos e automação. Os estudos tiveram como base de pesquisa uma residência na zona norte de Manaus/Amazonas, onde ocorre irregularidades no abastecimento de água nessa região, fazendo com que os moradores utilizem sistemas hidráulicos para abastecer seus reservatórios residenciais. Além disso, para que o estudo tivesse melhores resultados, foi aperfeiçoado um protótipo para uma melhor observação da operação automatizada do processo de abastecimento em escala menor. Através de um sistema hidráulico já existente na casa, o processo de automatização do mesmo se deu pela elaboração de um sistema elétrico com a função de acionamento da bomba através de um Arduino® e para alimentação dos sensores responsáveis por indicar o nível de água nos reservatórios. Foi desenvolvido um sistema de monitoramento simples e prático baseado em um princípio elétrico e um sistema hidráulico. Após os testes realizados, o modelo proposto neste trabalho se mostrou um sistema de abastecimento hidráulico simples e funcional, facilitando para o usuário o acompanhamento da operação de alimentação de água para a residência, o projeto foi elaborado utilizando elementos já existentes na residência afim de reduzir custos para o usuário, foi implantado um sistema autônomo no processo alimentação de água da residência, possibilitando assim a praticidade no acionamento da bomba hidráulica e o acompanhamento de níveis nos reservatórios da residência.

Palavras Chaves: Automatização. Sistema de Abastecimento de Água. Sistema Hidráulico. 


\section{INTRODUÇÃO}

Um dos principais desafios mundiais na atualidade é o atendimento à demanda por água de boa qualidade. $\mathrm{O}$ crescimento populacional, a necessidade de produção de alimentos e o desenvolvimento industrial devem gerar sérios problemas no abastecimento de água nos próximos anos [1].

Com o aumento da população, se elevou o consumo de água e o homementendeu a necessidade de executar grandes obras destinadas à capitação, transporte e armazenamento da água, passando então a desenvolver técnicas de tratamento interferindo assim no ciclo hidrológico natural e gerando um ciclo artificial. Nas últimas décadas o consumo de água cresceu duas vezes mais do que a população e a estimativa é que a demanda cresça ainda $55 \%$ até 2050. Mantendo os atuais padrões de consumo, em 2030 o mundo enfrentará um déficit no abastecimento de água de 40\% [2].

O Brasil é um dos países mais ricos em água doce do planeta, dotado de cerca de $12 \%$ de toda a água doce do planeta. Porém, sua distribuição não é uniforme em todo o território, a Amazônia é a região que detém a maior bacia fluvial do mundo, com o maior volume de água do planeta [3].

Em várias regiões do mundo a população enfrenta algum tipo de problema de água, seja ele de coleta ou abastecimento, em regiões secas no Brasil, a coleta de água é escassa, mas no país em que detém $12 \%$ da água doce do mundo[3], um grande obstáculo enfrentado é o de abastecimento de água em várias regiões, em regiões afastadas as famílias utilizam poços artesianos ou cisternas para o abastecimento de água. A ineficiência no abastecimento em residências urbanas também leva as famílias recorrerem à utilização de um sistema auxiliar de armazenagem e distribuição para garantir o fornecimento de água. Nesse caso o abastecimento é auxiliado por uma bomba hidráulica, tanto para a coleta de água em poços quanto para a alimentação de reservatórios.

Já é comumo uso de várias tecnologias nesses sistemas de abastecimento de água residencial, para garantir a eficiência na operação, que vão desde a instalação de sensores emreservatórios residenciais para o monitoramento do volume de água, até a automatização de acionamento de bombas hidráulicas para alimentação de reservatórios, o uso desses dispositivos se torna importante para garantir o perfeito funcionamento e evitar que ocorra a ausência de água nas residências. Esse trabalho se justifica, em razão da necessidade de se tornar um sistema de abastecimento de água residencial mais eficiente, automatizar sistemas de abastecimento é uma importante ferramenta para o monitoramento da operação, com a intenção de facilitar para o proprietário o acompanhamento da operação de abastecimento e assim identificar rapidamente algum problema que venha a surgir no decorrer da condução da água para o reservatório da residência.

A automatização desse sistema permitiria ao proprietário um maior controle da operação, com o acionamento da bomba caso um reservatório atingisse o volume mínimo e precisa-se ser alimentado por um outro reservatório ou poço.

\section{REVISÃO BIBLIOGRÁFICA}

De acordo com o [4], um sistema de abastecimento de água é concebido e projetado para atender pequenas populações ou grandes cidades, de acordo com características e porte de suas instalações. Caracteriza-se pela retirada da água da natureza, adequação de sua qualidade, transporte até os aglomerados humanos e fornecimento às populações em quantidade compatível com suas necessidades. $\checkmark$ Sistema de abastecimento individual

Problemas encontrados em algumas redes de distribuição pública, como a interrupção de abastecimento, pressão e variações da vazão de água nas redes de distribuição pública, levam os proprietários, a adotarem sistemas de abastecimento individuais como soluções provisórias ou definitivas. Algumas são soluções precárias para os centros urbanos, embora indicados para as áreas rurais onde a população é dispersa. Com a ineficiência do fornecimento de água pelo sistema público, adota-se a solução de recursos hídricos locais, como o sistema de poços (poço de lençol freático) ou poço artesiano.

O mais comum meio para bombeamento de água de poços, é a utilização de uma bomba centrifuga como podemos ver na figura 1 .

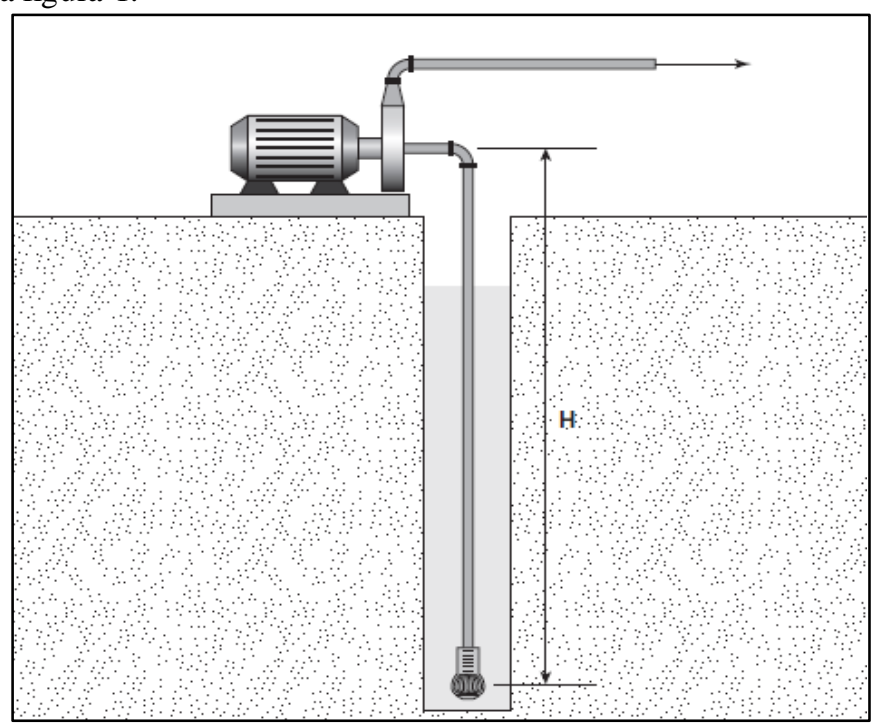

Figura 1: Bombeamento de água em poços através de bomba hidráulica.

Fonte: [5].

Esse sistema é adequado para poços pouco profundos, uma vez que a altura máxima de sucção de uma bomba centrifuga é teoricamente cerca de 10 metros. Mas na prática, devido a perdas nas tubulações, o valor máximo se situa na faixa de 7 a 8 metros [6].

\section{Reservatórios}

Os reservatórios têm a função de atender residências que enfrentam algum problema de abastecimento de água, manter o abastecimento no caso de paralização do fornecimento de água. Pode agregar um sistema de poços ou cisternas, além de caixas d água superiores funcionando como um reservatório extra, muito comum em áreas urbanas que enfrentam problemas de distribuição, onde costumam ser alimentados através de bombeamento de reservatórios inferiores [4].

São unidades hidráulicas de acumulação e pas sagem de água situados em pontos estratégicos do sistema de abastecimento residencial, de modo a atenderem as seguintes situações [5]:

- Garantir o fornecimento constante de água;

- Garantir a quantidade de água a ser usada;

- Garantir a vazão constante no sistema;

- Melhores condições de pressão no abastecimento. 


\section{Bombas Hidráulicas}

Bombas são maquinas geratrizes, com a finalidade de realizar o deslocamento de um liquido por escoamento, ela transforma o trabalho mecânico que recebe em energia [6]. A escolha por um outro sistema de abastecimento de água juntamente com o sistema de distribuição da concessionária provisório, ou permanente como ocorre em residências rurais onde não são atendidas pelo abastecimento público, requer o uso de motorbombas para o bombeamento da água até os reservatórios, inferior ou superior de acordo com as características e necessidades da residência. De acordo com [5] as bombas d’água são recomendadas para poços com pouca profundidade, muito comum em sistemas de abastecimento hidráulico de água em residenciais.

\section{Bombas centrífugas}

Bombas centrifugas são maquinas hidráulicas que tem como princípio de funcionamento a transferência de energia mecânica obtido através de palhetas e impulsores que giram no interior da carcaça (corpo da bomba), bombeando o fluido do centro para a periferia da carcaça. No seu interior a energia de velocidade é transformada em energia de pressão, o que possibilita o liquido alcançar o ponto final de recalque. A energia transformando o fluido em energia potencial (energia de pressão), é sua principal característica [6]. É o equipamento mais utilizado para bombear liquido em saneamento básico, irrigação de lavouras, edifícios residenciais e industrias. Ideal para instalações de bombeamento de água em residências, abastecendo reservatórios com o bombeamento a partir de cisternas ou poços como mostra a Figura 2.

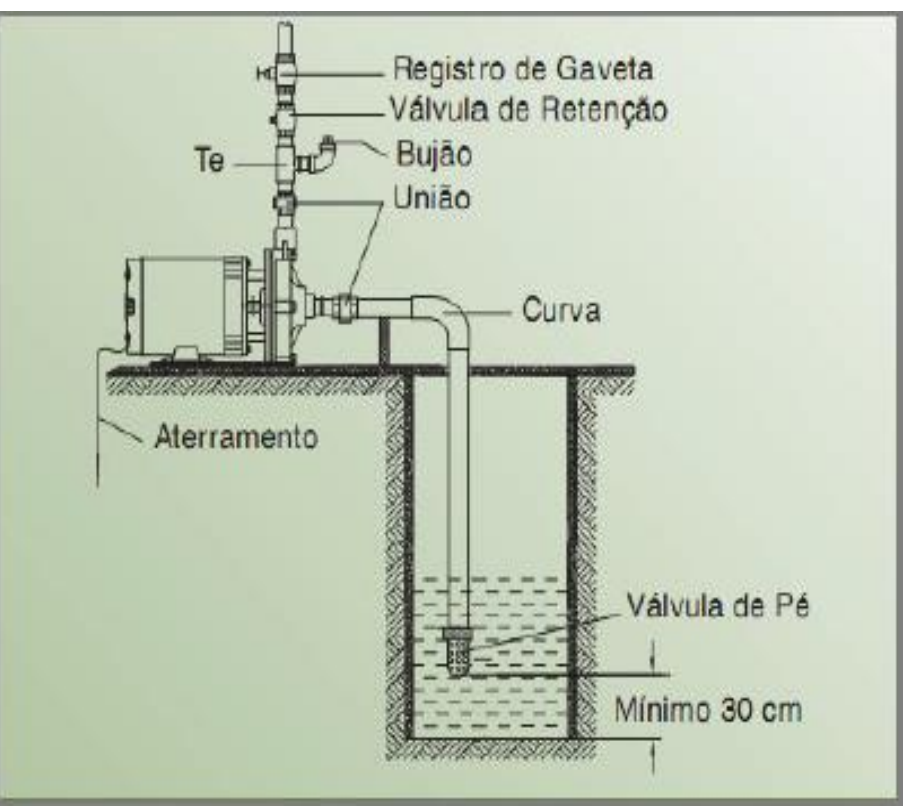

Figura 2: Esquema de instalação com bomba centrifuga de simples estágio.

Fonte: [7].

\section{Altura geométrica}

Também chamado de altura estática, são desníveis geométricos a serem vencidos pelo fluxo no sistema, e pode ser dividido em dois estágios; altura de sucção e altura de recalque.

\section{Altura de sucção}

A altura de sucção é a diferença entre o nível dinâmico da captação e o bocal de sucção da bomba, quando a água na captação está submetida a pressão atmosférica [8]. No entanto a altura de sucção não é limitada somente pela pressão atmosférica local, mas também, pelas perdas de carga, atrito e pela turbulência ao longo da sucção no interior da bomba. Em bombas centrifugas normais, instaladas ao nível do mar e com fluido bombeado a temperatura ambiente, esta altura não pode exceder 8 metros de coluna d’água (8 mca).

\section{Altura de recalque}

É a altura entre o bocal de sucção da bomba e o ponto de maior elevação do fluido, até o reservatório [8], onde a cessão de energia ao fluido constituído pelas canalizações vence o desnível geométrico entre a bomba hidráulica e o reservatório superior.

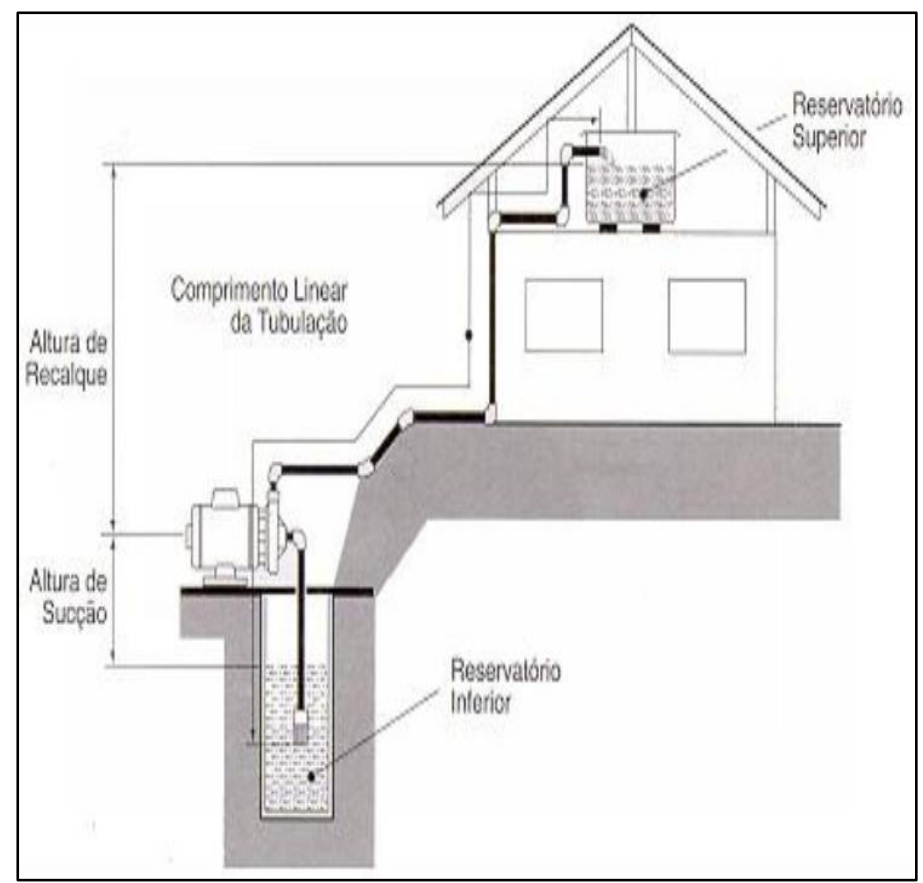

Figura 3: Esquema representando altura de sucção e altura de recalque.

Fonte: [8].

\section{$>\quad$ Altura manométrica}

Éa soma das alturas de sucção e recalque, definida como altura geométrica. É a energia hidráulica que a bomba deverá transmitir ao líquido para transportar a vazão do reservatório inferior ao reservatório superior da ins talação, vencendo o desnível geométrico, perdas de cargas por atritos e choques em conexões e tubulações, além da diferença de pressões nos reservatórios [8].

\section{$>\quad$ Sensores de nível}

Os sistemas hidráulicos automáticos têm se tornado cada vez mais comuns nas instalações residenciais urbanas ou em propriedades rurais, cada vez mais esses sistemas têm se tornado fundamentais. Existem diversas configurações para sistemas de controle de nível, sendo o mais comumdeles o uso de dois sensores de nível para acionar uma bomba d’água [9]. De acordo com [9] 
basicamente qualquer tipo de automação, pode realizar: detecção, transferência e avaliação de sinais.

Esse sistema possui um baixo custo de instalação, com diversos tipos de sensores no mercado de baixo custo e longa duração. Os sistemas têm a também a facilidade de ser construído de forma caseira, e ser adaptado de acordo com as características de cada sistema de abastecimento.

Existem diversas opções de sensores de acordo com [9]:

- $\quad$ Boias magnéticas;

- Boia lateral;

- $\quad$ Sensores de imersão;

- Medição através de sensores de ultra-som.

Os sensores se tornaram importantes ferramentas nos diversos sistemas de abastecimento, tanto em grandes escalas, como na indicação de níveis de reservatórios, como em pequenos reservatórios domésticos.

Importante para o funcionamento e segurança do abastecimento, é responsável por indicar excessos ou baixo nível de água nos reservatórios. Sendo inclusive usados em acionamentos de bombas hidráulicas.

\section{Automação}

Nas últimas décadas, com o avanço na engenharia eletrônica, foi possível o desenvolvimento de computadores e equipamentos sofisticados para serem utilizados emautomação dos sistemas de abastecimento de água e esgoto sanitário. Como a automação consiste na substituição da ação humana pela mecânica ou por dispositivos criado pelo homem é de fundamental importância o conhecimento detalhado do funcionamento do sistema hidráulico e processos dos equipamentos eletromecânicos [10]. Utilizando-se de elementos como CLP ou Arduino®, sendo uma das principais ferramentas no campo da automação.

- Automação de sistemas de abastecimento de água A automação tem como objetivo independendo da característica do sistema de abastecimento, cumprir as tarefas [10]:

- $\quad$ Controle de energia elétrica;

- Integração operacional;

- $\quad$ Centralização operacional;

- Fazer o acompanhamento das operações de captação e distribuição de água;

- Possibilitar a operação e acompanhamento à distância, sem a necessidade de deslocamento até o ponto monitorado;

- $\quad$ Acompanhar o monitoramento em tempo real de variações de níveis nos reservatórios;

- Antecipar ao usuário, possíveis falhas ou qualquer problema que venha a surgir durante a operação de abastecimento ou dis tribuição.

\section{MATERIAIS E MÉTODOS}

\section{III.1 METODOLOGIA DA PESQUISA}

Este trabalho foi elaborado com base em uma pesquisa experimental, partindo de uma pesquisa bibliográfica em diversas literaturas como revistas especializadas, artigos, teses, dissertações, monografias, manuais dentre outros, como intuito de avaliar possíveis soluções já existentes para o problema descrito, as sim como a elaboração de ummodelo que poss a se utilizar destas soluções de forma que solucione os problemas do nosso objeto de estudo.

De acordo com [11] a pesquisa bibliográfica é "um apanhado geral sobre os principais trabalhos já realizados, revestidos de importância, por serem capaz de fornecer dados atuais e relevantes relacionados com o tema", com o objetivo de buscar trabalhos que abordem o mesmo problema e as soluções elaboradas, permitindo nos aprofundar mais no tema e atender aos objetivos propostos com esta pesquisa.

Em relação à pesquisa experimental [12], afirma que a mesma constitui-se da elaboração de um objeto de estudo, da seleção das variáveis capazes de influencia-lo e definir formas de controle e observação dos efeitos produzidos pela variável no objeto de estudo, pois temos como meta o estudo de uma residência e a elaboração de métodos para solucionar o problema de falta de água.

Após apurar as bibliografias necessárias, fizemos um levantamento dos dados do objeto de estudo, sendo uma residência situada no bairro Manôa na cidade de Manaus, AM, composto por uma residência de 2 andares, sendo ocupada por 4 moradores. A água potável é fornecida pelo sistema público de distribuição de Manaus e é utilizado para consumo direto, higiene pessoal, limpeza da residência e preparo de alimentos. A água é utilizada em três banheiros, uma área de serviço e em torneiras para lavagem de utensílios domésticos.

\section{III.2 COLETA DE DADOS}

A coleta de dados partiu de um levantamento dos elementos já instalados na residência, com o intuído de realizar cálculos de dimensionamento e certificar se os mesmos atendem à demanda de forma segura.

Foi realizado a medição da altura em relação a bomba hidráulica e cisterna que alimenta a residência, bem como o comprimento total do encanamento, com o propósito de dimensionar se a bomba hidráulica possui a potência adequada. Anotamos os valores em tabelas para consulta posteriores, pesquisamos a amperagem de trabalho da bomba no catálogo da fabricante e dimensionamos o cabo elétrico e o disjuntor utilizado para garantir a segurança do sistema.

Realizou-se o dimensionamento dos elementos de monitoramento e automatização do sistema, como a determinação das fontes de alimentação baseadas na demanda do sistema e o requerimento mínimo para o funcionamento de elementos como o e as lâmpadas LED.

Aplicamos em um protótipo em menor escala, com o propósito de facilitar o estudo dos sensores empregados e do programa de automação em situações controladas. Anotamos os resultados obtidos emtodos os testes e diagnos ticamos os pos síveis erros na construção do protótipo, para posterior aplicação no objeto de estudo.

Realizado os testes com diferentes configurações de posicionamento dos sensores de nível do tipo boia, buscando garantir que não ocorram falhas durante o funcionamento da bomba. Foram criadas situações de falta de abastecimento de água no sistema e observou-se como o sistema reage, e também evitar que haja desperdício de água. Detectou-se os possíveis problemas envolvidos com a programação do Arduino ${ }^{\circledR}$, e observamos quais eram os erros mais comuns. Utilizou-se um programa que dispensasse atuação do usuário, e is to requer a confiabilidade no 
programa, além optar pela utilização de um programa simples e eficiente.

Observou-se também se os medidores de nível de água estavam funcionando como o desejado, marcando corretamente o nível das cisternas, ou se apresentavam falhas de contato elétrico. Foi checado se os LED estavam recebendo energia elétrica e iluminando corretamente. Foi feita uma vistoria geral para detectar se todos os cabos elétricos estavam devidamente is olados e se não havia perigo de choques elétricos.

\section{RESULTADOS E DISCUSSÕES}

\section{IV.1 APRESENTAÇÃO DOS RESULTADOS COLETADOS}

Os dados obtidos na medição da residência se encontram compatíveis, podendo ser utilizados no projeto proposto. A potência da bomba hidráulica instalada mostrou-se superior a desejada no cálculo, aumentado a vazão desejada de $0,5 \mathrm{~m}^{3} / \mathrm{s}$ para $8,5 \mathrm{~m}^{3} / \mathrm{s}$. De acordo com a tabela (2) que veremos a diante neste trabalho, a tubulação utilizada pela residência não é ideal para a potência da bomba hidráulica, sendo necessário a utilização de uma tubulação de 2 polegadas.

O dimensionamento dos elementos presentes no sistema da residência mostra que estão de acordo como que descreveu [5] sobre o sistema de abastecimento individual, onde se adota um sistema com elementos e soluções provisórias ou definitivas, com a utilização de tubulações e a escolha de bombas d’água sem o devido dimensionamento.

Durante os testes em protótipo, observou-se que a garrafa PET de 2L utilizada para representar a cisterna inferior possui um volume demasiadamente pequeno, e a potência da bomba hidráulica retirada da máquina de lavar utilizada para simular o protótipo é muito superior, o que dificultou a analise o ciclo. Desta forma, a utilização da cisterna inferior ter o dobro de volume da cisterna superior, quando for implantado este projeto na residência de estudo, se faz necessário.

Os elementos adicionais que foram utilizados para a elaboração do protótipo foram comprados seguindo os valores calculados conforme descrito neste trabalho. A lista dos Itens para a compra está listada no Anexo A e possui a quantidade necessária dos elementos, bem como a especificação dos elementos. Durante as simulações no protótipo o controle do processo de ativação da bomba hidráulica por meio dos sensores de nível se provaram funcionais. Foi provado o funcionamento dos LEDs de detecção de nível da água, mesmo na cisterna inferior onde a vazão de água era muito superior.

Durante o experimento com o sensor de nível não se detectou nenhum problema na ativação e no desligamento da bomba hidráulica. A autonomia do sistema ocorreu com segurança e a atuação do usuário não se fez necessária, mesmo em situações que simulamos a falta de água.

Devido a problemas na distribuição de água pela concessionária responsável, projetou-se um sistema de armazenamento de água, utilizando-se duas cisternas, uma localizada na parte abaixo, utilizada para armazenamento da água vinda da rede de distribuição pública, a bomba hidráulica para transportar a água, e uma segunda cisterna localizada em uma base elevada a 4,00m, responsável por suprir a residência. Além disso, a utilização de dispositivo para controle e automatização deste sistema e testa em um protótipo.

Foi feito um levantamento a respeito dos materiais necessários para o projeto, tais como: Cisterna de água; Bomba hidráulica; Placa Arduino ${ }^{\circledR}$ Uno; Conjunto de Sensor de nível caseiro; Fita de LEDs; Relé 110/220V.

Após a apuração dos materiais, iniciou-se a parte de dimensionamento dos mesmos para que seja aplicado na residência descrita anteriormente. A fim de melhor compreensão, eles serão divididos nas respectivas áreas: hidráulica, elétrica, automatização e protótipo.

\section{IV.2 O SISTEMA HIDRÁULICO}

\section{IV.2.1 CISTERNA}

O sistema hidráulico possui em sua instalação a cisterna Fortlev 1500 litros de polietileno para a cisterna superior, enquanto para a cisterna inferior utilizamos uma de mesma marca, porém com 3000 litros de capacidade. Foi selecionado com o dobro de volume entre ambas com o intuito de que sempre haja água disponível na cisterna inferior e evitar que a cisterna superior fique sem água quando solicitado. De acordo com [13], para uma residência de até 5 pessoas, a cisterna inferior deve possuir $3 / 5$ de volume adicional para um consumo de dois dias. Dessa forma, para 1500L, deve haver um adicional de 900L, resultando em 2400L. Sugere-se a obtenção de uma cisterna de 3000L.

Tais especificações vão de acordo com o [14], que ressalta que o reservatório inferior será alimentado pela rede de distribuição e alimentará o reservatório superior por meio de um sistema de recalque (conjunto moto-bomba), e em seguida alimentará os pontos de consumo da residência por gravidade.

\section{IV.2.2 BOMBA HIDRÁULICA}

Para seleção da bomba hidráulica utilizou-se o manual da fabricante de bombas Meka. Segundo o [15], para fazer a seleção adequada de uma bomba hidráulica são necessários estipular uma vazão para o sis tema e calcular os desníveis de sucção, os desníveis de recalque, as perdas de atrito na tubulação e no recalque e as perdas de atrito nas conexões hidráulicas, representado pelas seguintes equações:

$$
\text { ATM }=H+h+\text { Perdas }
$$

Onde ATM se refere a altura manométrica total do sistema, onde: Hé a altura medida de sucção, e é medida da ponta do encanamento até a entrada da bomba;

h é a altura de recalque, sendo medida da altura da saída da bomba até a entrada da cisterna;

Perdas de cargas em conexões, cujo os valores obtidos com as equações a seguir.

Os valores de perdas são calculados de acordo com as seguintes equações:

Perdas sucção $=\frac{(\text { Altura Sucção }+ \text { Perdas Conecções }) \cdot \text { Perda atrito }}{100}$

Os valores das perdas de altura de sucção são obtidos medindo a tubulação de sucção, que corres ponde a tubulação que vai da saída da cisterna inferior até a entrada da bomba hidráulica, somando perdas de cargas em conexões na tubulação, que corresponda aos valores obtidos na tabela abaixo na tabela 1, e levam em conta o diâmetro da tubulação e as conexões presentes no sistema. 
Farias \& Silva, ITEGAM-JETIA. Vol. 03, № 10, pp.104-115. Junho. 2017.

Tabela 1: Perdas de carga em conexões.

\begin{tabular}{|c|c|c|c|c|c|c|c|c|c|c|c|c|c|}
\hline \multicolumn{14}{|c|}{ Em metros de Tubulação Equivalente } \\
\hline \multirow{2}{*}{ Conexōes } & \multicolumn{13}{|c|}{ Diáme tro das Conexōes } \\
\hline & $3 / 4^{\prime \prime}$ & $1^{\prime \prime}$ & 1.1/4" & $1.1 / 2^{\prime \prime}$ & $2^{\prime \prime}$ & $2.1 / 2^{\prime \prime}$ & 3" & $4^{\prime \prime}$ & $5^{\prime \prime}$ & $6^{\prime \prime}$ & $8^{\prime \prime}$ & $10^{\prime \prime}$ & $12^{\prime \prime}$ \\
\hline Reg. Gaveta & 0,10 & 0,12 & 0,18 & 0,20 & 0,28 & 0,34 & 0,46 & 0,65 & 0,83 & 1,10 & 1,50 & 1,80 & 2,37 \\
\hline Reg. Globo & 5,00 & 6,80 & 9,70 & 11,80 & 16,00 & 20,00 & 26,00 & 37,00 & 48,00 & 60,00 & 83,00 & 103,00 & 135,00 \\
\hline Válv. Retenção & 1,10 & 1,50 & 2,10 & 2,50 & 3,40 & 4,30 & 5,50 & 7,70 & 10,20 & 12,60 & 17,60 & 21,70 & 28,60 \\
\hline Curva $90^{\circ}$ & 0,30 & 0,40 & 0,60 & 0,70 & 1,00 & 1,20 & 1,50 & 2,00 & 2,80 & 3,50 & 4,90 & 6,00 & 7,90 \\
\hline Cotovelo $45^{\circ}$ & 0,30 & 0,40 & 0,50 & 0,60 & 0,90 & 1,10 & 1,40 & 1,90 & 2,50 & 3,20 & 4,40 & 5,40 & 7,10 \\
\hline Cotovelo 90 Tee & 0,60 & 0,80 & 1,10 & 1,30 & 1,80 & 2,20 & 2,90 & 4,00 & 5,20 & 6,50 & 9,00 & 11,30 & 14,80 \\
\hline Válv. De Pé & 10,80 & 14,90 & 21,00 & 26,00 & 35,00 & 44,00 & 57,00 & 79,00 & 100,00 & 130,00 & 180,00 & 225,00 & 300,00 \\
\hline
\end{tabular}

Fonte: [ ]. Catalogo de Bombas - MEKA (2010, p. 63)

Os valores das perdas de altura de sucção são obtidos medindo a tubulação de sucção, que corresponde a tubulação que vai da saída da cisterna inferior até a entrada da bomba hidráulica, somando perdas de cargas em conexões na tubulação, que corresponda aos valores obtidos na tabela 2 a tabela em anexo $\mathrm{C}$, e levam em conta o diâmetro da tubulação e as conexões presentes no sistema. A perda de carga por atrito é um percentual obtido em tabela levando em conta a vazão desejada e o diâmetro da tubulação, de acordo com a tabela 2 :

Tabela 2: Perdas de cargas por atrito em tubulação.

\begin{tabular}{|c|c|c|c|c|c|c|c|c|c|c|c|c|c|}
\hline \multicolumn{14}{|c|}{$\begin{array}{r}\text { Tabela de Perda de Carga por Atrito en } \\
\qquad \text { Para cada } 100 \text { metros (Ferro ou Aço) }\end{array}$} \\
\hline \multirow{2}{*}{ Vazão $\mathrm{m} 3 / \mathrm{h}$} & \multicolumn{13}{|c|}{ Diámetro da Tubulaçâo } \\
\hline & $3 / 4^{\prime \prime}$ & $1 "$ & $1.1 / 4^{\prime \prime}$ & $1.1 / 2$ & 2 " & 2.1/2" & $3^{*}$ & $4^{*}$ & 5 & $6^{\prime \prime}$ & $8^{-1}$ & $10^{-\prime}$ & $12=$ \\
\hline 0,5 & 2,00 & 0,70 & 0.20 & & & & & & & & & & \\
\hline 1,0 & 7,50 & 2,70 & 0,75 & 0,22 & 0,08 & & & & & & & & \\
\hline 1,5 & 16,00 & 6,00 & 1,60 & 0,50 & 0,17 & & & & & & & & \\
\hline 2,0 & 27,00 & 10,00 & 2,70 & 0,80 & 0,28 & 0,07 & & & & & & & \\
\hline 2,5 & 35,00 & 16,00 & 4,50 & 1,40 & 0,40 & 0,12 & & & & & & & \\
\hline 3,0 & 58,00 & 21,50 & 6,00 & 1,80 & 0,60 & 0,16 & 0,05 & & & & & & \\
\hline 3,5 & 80,00 & 26,00 & 8.00 & 2,40 & 0,80 & 0.22 & 0,08 & & & & & & \\
\hline 4,0 & 100,00 & 37,00 & 10,00 & 3,00 & 1,05 & 0,27 & 0,10 & & & & & & \\
\hline 4,5 & & 45,00 & 12,00 & 3,70 & 1,30 & 0,32 & 0,12 & & & & & & \\
\hline 5,0 & & $55, \infty$ & 15,50 & 4,70 & 1,60 & 0,42 & 0,15 & & & & & & \\
\hline 5,5 & & 65,00 & 18,00 & 5,50 & 2,00 & 0,50 & 0,17 & & & & & & \\
\hline 6,0 & & 80,00 & 22,00 & 6,60 & 2,20 & 0,60 & 0,20 & 0,07 & & & & & \\
\hline 6,5 & & 95,00 & 25,00 & 7,50 & 2,40 & 0,70 & 0,26 & 0,08 & & & & & \\
\hline 7,0 & & & 29,00 & 8,30 & 3,00 & 0,80 & 0,28 & 0,10 & & & & & \\
\hline 7,5 & & & 35,00 & 11,00 & 3,50 & 0,90 & 0,30 & 0,12 & & & & & \\
\hline 8,0 & & & 37,00 & 11,50 & 3,90 & 1,00 & 0,35 & 0,13 & & & & & \\
\hline 8,5 & & & 38,00 & 13,00 & 4,50 & 1,20 & 0,40 & 0,16 & & & & & \\
\hline 9,0 & & & 40,00 & 14,00 & 4,80 & 1,25 & 0,45 & 0,18 & & & & & \\
\hline 9,5 & & & 50,00 & 15,00 & 5,10 & 1,40 & 0,47 & 0,19 & & & & & \\
\hline A & & & $e n$ & $m m$ & $=-m$ & $\Leftrightarrow$ en & nen & a ma & 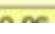 & & & & \\
\hline
\end{tabular}

Fonte: [ 15].

De forma, semelhante perda altura de sucção, as perdas de altura de recalque são calculadas de forma semelhante, utilizando os valores da altura de recalque.

Perdas Recalque $=\frac{(\text { Altura recalque } \cdot \text { Perdas Cargas }) \cdot \text { Perda atrito }}{100}$ (3)

No sistema estudado, nós temos os seguintes valores:

- Vazão mínima: $0,5 m^{3} / \mathrm{h}$
Sucção:

- $\quad$ Altura de sucção: $0,5 \mathrm{~m}$

- Tamanho da tubulação: $0,7 \mathrm{~m}$

- 2 curvas de 1 polegada em $90^{\circ}$

- 1 registro de 1 polegada

Recalque

- Altura de recalque: $5,05 \mathrm{~m}$

- Tamanho da tubulação $10,53 \mathrm{~m}$

- 8 curvas 1 polegada em $90^{\circ}$ 
Optou-se por utilizar uma vazão de referência de $0,5 \mathrm{~m}^{3} / \mathrm{h}$ por questões de segurança, uma vez que não precisamos ter uma vazão muito alta para o nosso sistema. Os diâmetros da tubulação correspondem aos valores obtidos com a medição da rede já existente.

Utilizando as tabelas (1) e (2) de referência e as equações Eq. (1), (2), (3), temos:

$$
\begin{gathered}
\text { Perdas sucçã } o=\frac{[0,7+(2 \cdot 0,40)] \cdot 0,70}{100}=0,0105 \mathrm{~m} \\
\text { Perdas recalque }=\frac{[10,53+(8 \cdot 0,40)] \cdot 0,70}{100}=0,09611 \mathrm{~m} \\
A T M=5,05+0,5+0,0105+0,09611 \\
A T M=5,65661 \mathrm{~m}
\end{gathered}
$$

Encontra-se então, que a altura manométrica total é de aproximadamente 5,7 metros. Levando em conta uma vazão mínima de $0,5 \mathrm{~m}^{3} / \mathrm{h}$, devemos calcular a potência da bomba, que é definida pela equação:

$$
P=\frac{\delta \cdot Q \cdot H_{t}}{75 \eta t}
$$

Onde temos:

Q: vazão em $\mathrm{m}^{3} / \mathrm{s}$

$H_{t}$ : Altura manométrica total (ATM)

$\eta_{t}:$ Rendimento total $(\%)$

$\delta$ : Peso especifico da água $\left(\mathrm{Kg} / \mathrm{m}^{3}\right)$

$$
\begin{gathered}
P=\frac{1000 \cdot 0,00013 \cdot 5,65661}{75 \cdot 0,98} \\
P=0,010 C V
\end{gathered}
$$

Com base nos valores obtidos, encontra-se uma grande

\begin{tabular}{|c|c|c|c|c|c|c|c|c|c|c|c|c|c|c|c|c|}
\hline \multirow{3}{*}{ Modelo } & \multirow{3}{*}{ CV } & \multirow{2}{*}{\multicolumn{2}{|c|}{ Bocais }} & \multicolumn{13}{|c|}{ Altura Monométrica Total (MCA) } \\
\hline & & & & 02 & 03 & 04 & 05 & 06 & 07 & 08 & 09 & 10 & 12 & 14 & 16 & 18 \\
\hline & & Sucça 0 & Recalque & \multicolumn{13}{|c|}{ Vazáo em metros cúbicos por hora } \\
\hline $\mathrm{VH}-\mathrm{A}$ & $1 / 3$ & \multirow{6}{*}{$\begin{array}{l}1.1 / 2^{\prime \prime} \\
\text { Cola }\end{array}$} & \multirow{6}{*}{$\begin{array}{l}1.112^{\prime \prime} \\
\text { Cola }\end{array}$} & 11 & 10,5 & 9,9 & 9,1 & 8,5 & 7,5 & 6,5 & 5,5 & 4,4 & 1 & & & \\
\hline VH.2 & $1 / 2$ & & & 12,6 & 11,5 & 10,8 & 9,5 & 9,1 & 8,5 & 7,6 & 6,7 & 5,6 & 2,5 & & & \\
\hline VH3 3 & $3 / 4$ & & & 14,6 & 14,2 & 13,8 & 13,1 & 12,6 & 11,5 & 10,5 & 9,5 & 9 & 7,5 & 3,5 & & \\
\hline VH4 & 1 & & & 16 & 15,6 & 15,1 & 14,5 & 14,1 & 13,4 & 12,8 & 12 & 11 & 8 & 4 & & \\
\hline VH.5 & 1,5 & & & 21,8 & 21 & 20,5 & 19,9 & 19 & 18 & 17,2 & 16,2 & 15,2 & 13 & 10 & 6 & 1,2 \\
\hline VH6 & 2 & & & 25 & 24,5 & 24 & 23 & 22 & 21 & 20 & 19 & 17 & 15 & 12 & 8 & 3 \\
\hline
\end{tabular}
variedade de modelos para escolher no catálogo. Na residência que foi elaborado o estudo, está instalada uma bomba de modelo Meka VH-1 de 1/3 CV residência.

Na tabela 3, vemos os dados da bomba instalada na

Tabela 3: Tabela de Rendimentos da bomba.

Fonte: [15].

Nota-se que para a altura manométrica da residência estudada é a vazão é de $8,5 \mathrm{~m}^{3} / \mathrm{h}$.

Observa-se que a bomba é capaz de satisfazer o sistema estudado, embora a vazão se torne um tanto elevada. E estando de acordo com [5] que ressalta que as bombas d’água são recomendadas para poços com pouca profundidade, muito comum em sistemas de abastecimento hidráulico de água em residências.

\section{IV.3 O SISTEMA ELÉTRICO}

O sistema elétrico para o acionamento da bomba através do Arduino ${ }^{\circledR}$ e para alimentação dos sensores é bastante simples. $\mathrm{O}$ acionamento da bomba foi feito utilizando-se de um relé de 110/220 V acionado por 5V, como observado na figura 1. 


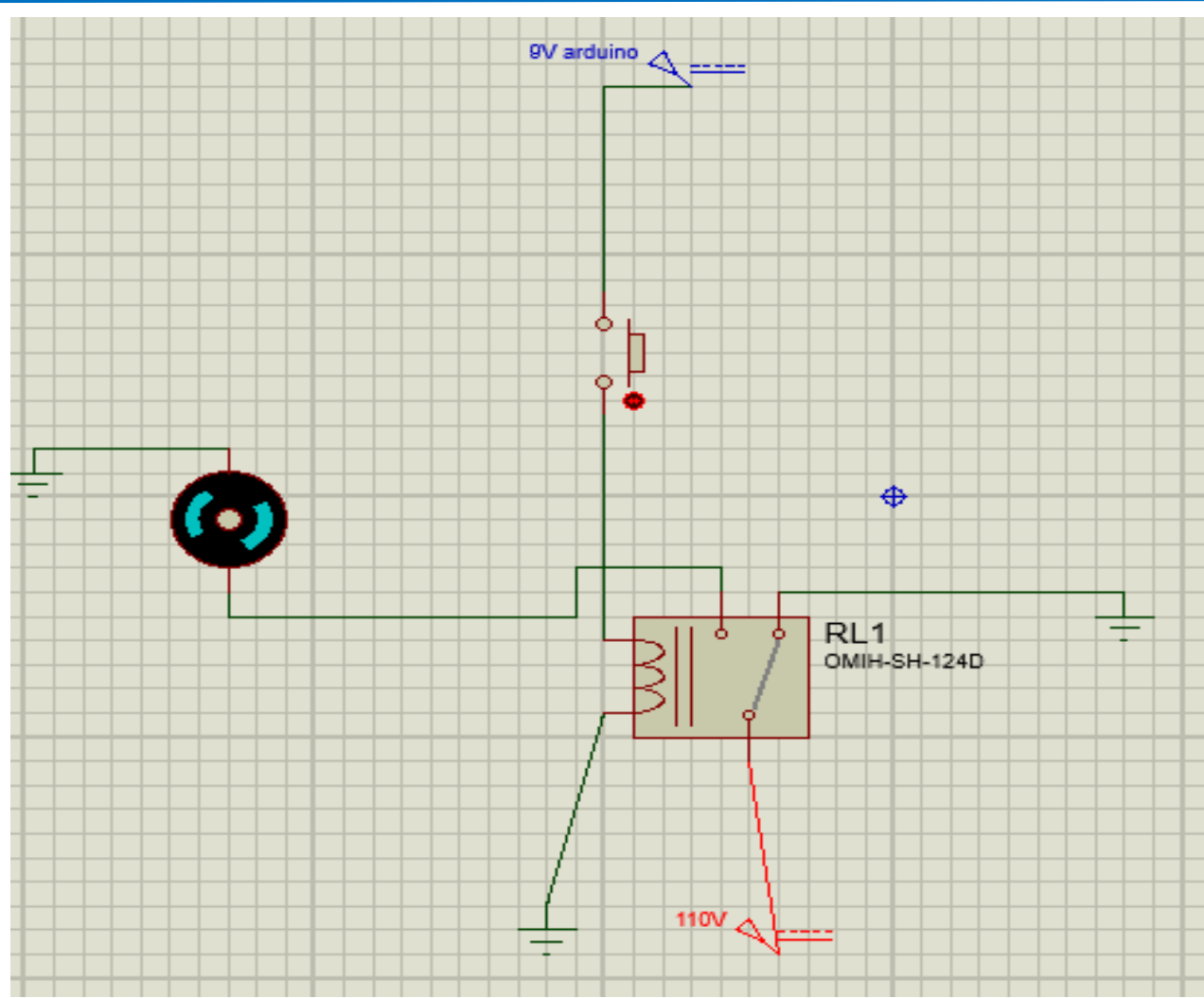

Figura 4: Esquema Elétrico.

Fonte: Autores, (2016).

Dessa forma, como observado na figura o relé é alimentado pela rede elétrica de $110 \mathrm{~V}$, e na entrada da bobina do relé liga-se ao Arduino®, enquanto na entrada normalmente aberta conecta-se na bomba, de forma que quando o Arduino ${ }^{\circledR}$ envia o sinal elétrico ativa o relé, fechando o chaveamento da bobina, ativando a passagem de corrente elétrica, que, por sua vez ativa a bomba. O relé utilizado é um relé Goodsky GS-SH-205t, e possui as seguintes características, conforme a tabela 4 :

Tabela 4: Datasheet do relé.

\begin{tabular}{c|c|c|c}
\hline Load Type & GS (D) & GS (T) & GS (L) \\
\hline \multirow{2}{*}{ Rated Load (Resistive) } & 1A 120VAC & 1A 120VAC & 1A 120VAC \\
\cline { 2 - 4 } & $2 \mathrm{~A} 24 \mathrm{VDC}$ & $2 \mathrm{~A} 24 \mathrm{VDC}$ & $2 \mathrm{~A} 24 \mathrm{VDC}$ \\
\hline Rated Carrying Current & $2 \mathrm{~A}$ & $2 \mathrm{~A}$ & $2 \mathrm{~A}$ \\
\hline \multirow{2}{*}{ Max. Allowable Voltage } & AC 120V & AC 120V & AC 120V \\
\cline { 2 - 4 } & DC 24V & DC 24V & DC 24V \\
\hline Max. Allowable Current & $2 \mathrm{~A}$ & $2 \mathrm{~A}$ & $2 \mathrm{~A}$ \\
\hline \multirow{2}{*}{ Max. Allowable Power Force } & $120 \mathrm{VA}$ & $120 \mathrm{VA}$ & $120 \mathrm{VA}$ \\
\cline { 2 - 4 } & $48 \mathrm{~W}$ & $48 \mathrm{~W}$ & $48 \mathrm{~W}$ \\
\hline Min. Switching Load & DC 1V, 1mA & DC 1V, 1mA & DC 1V, 1mA \\
\hline Contact Material & Ag Alloy & Ag Alloy & Ag Alloy \\
\hline Contact Form & DPDT & DPDT & DPDT \\
\hline
\end{tabular}

Fonte: [16].

Com base nos dados da tabela, observa-se a faixa de operação do relé suporta até $120 \mathrm{~V}$. Por razões de segurança, a bomba está ligada a um disjuntor, cuja potência é calculada pela lei da Potência:

$$
\mathrm{I}=\mathrm{P} / \mathrm{V}
$$

Onde I se refere a corrente elétrica, $\mathrm{P}$ é a potência da bomba em watts e $\mathrm{V}$ é a voltagem.
Temos que:

$$
\begin{aligned}
& I=\frac{245,1}{127} \\
& I=1,92 \mathrm{~A}
\end{aligned}
$$

Em cima deste valor obtido aplicamos $20 \%$ acima deste valor, para aumentar a segurança do sistema, então obtivemos I= 2,28A. Para determinar o diâmetro do cabo, utilizou-se de uma tabela 5 de cabos. 
Farias \& Silva, ITEGAM-JETIA. Vol. 03, № 10, pp.104-115. Junho. 2017.

Tabela 5: Tabela de seleção da secção do cabo.

\begin{tabular}{|c|c|c|c|c|c|}
\hline Corrente & \multicolumn{5}{|c|}{ Seção do cabo ( $\mathrm{mm} 2)$ de acordo com comprimento do cabo (metros) } \\
\hline $\begin{array}{l}\text { Consumo } \\
\text { (amperes) }\end{array}$ & Até $1 \mathrm{~m}$ & $1 \mathrm{~m}$ a $2 \mathrm{~m}$ & 2 a $3 \mathrm{~m}$ & 3 a $5 \mathrm{~m}$ & 5 a $7 \mathrm{~m}$ \\
\hline 1 a 20 Aa & 4.00 & 4.00 & 4.00 & 4.00 & 6.00 \\
\hline 20 a $30 \mathrm{Aa}$ & 4.00 & 4.00 & 6.00 & 6.00 & 13.30 \\
\hline 30 a $40 \mathrm{~A}$ & 4.00 & 4.00 & 6.00 & 13.30 & 13.30 \\
\hline 40 a $60 \mathrm{~A}$ & 6.00 & 6.00 & 13.30 & 13.30 & 13.30 \\
\hline
\end{tabular}

Fonte: [17]

De acordo com a tabela e considerando a distância entre a bomba e o disjuntor é de $2 \mathrm{~m}$, utilizamos um cabo de $4.00 \mathrm{~mm}$. A fonte utilizada deve possuir uma potência suficiente para o funcionamento dos LEDs indicadores de nível. Selecionou-se um LED que trabalha com uma voltagem de $12 \mathrm{~V}$ devido a praticidade de não ser necessário a construção de um sistema elétrico, o qual necessitaria dimensionar os resistores entre outros empecilhos, e a fonte utilizada foi uma fonte externa de 12V.O funcionamento do medidor de nível será descrito adiante no trabalho. Em relação ao Arduino®, segundo o seu datasheet presente do anexo g, observamos que se recomenda a utilização de uma fonte de $6 \mathrm{~V}$. Optamos por utilizar uma fonte de 9 volts, para alimentar tanto o Arduino ${ }^{\circledR}$, quanto o sensor de nível do tipo boia, pois estes também necessitam de energia elétrica para funcionamento.

\section{IV.4 AUTOMATIZAÇÃO}

Com o objetivo de tornar o sistema dinâmico e independente da deficiência abastecimento do sistema público, foi desenvolvido um sistema de monitoramento do nível de água, e um sistema de automatização do mesmo.

\section{IV.4.1 SISTEMA DE MONITORAMENTO}

Para o sistema de monitoramento, desenvolveu-se um sistema simples baseado em um princípio elétrico. Ele consiste em um conjunto de fios elétricos ligados emparalelo e são alimentados por uma fonte de $12 \mathrm{~V}$. Estes fios são interligados seguimentos da fita de LED descrita anteriormente, e representam o nível de água presente na cisterna, sendo divididos em 4 níveis e um nível comum. Cada nível possui um segmento de fita de LED composto por 3 LEDs que é aceso quando a água atinge determinado nível. Cada nível é ligado a uma fonte de energia em paralelo e, conforme o nível da água sobe e entra em contato como parafuso, este acaba fechando contato elétrico. Para o funcionamento correto, é necessário que pelo menos um dos fios esteja sempre em contato com a água, para garantir a presença de corrente elétrica no conjunto em paralelo, sendo este categorizado como o fio comum. Cada nível é possui um parafuso inox de 1 polegada, responsável por realizar o contato elétrico com a água. Cada parafuso inox é montado em uma estrutura de canos de PVC, de forma que apenas o parafuso estará em contato com a água. O sistema elétrico funciona de forma semelhante a imagem da figura 5 .

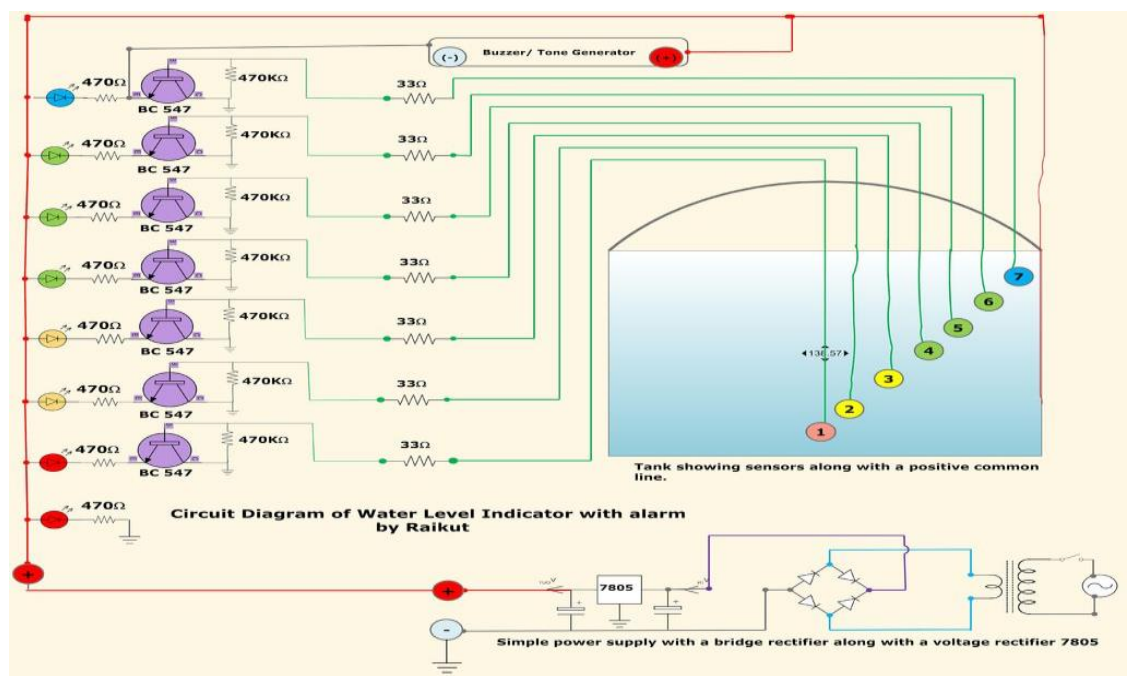

Figura 5: Funcionamento do esquema elétrico do sensor.

Fonte: Autores, (2016). 


\section{IV.5 O SISTEMA DE AUTOMATIZAÇÃO}

Para automatizar o sistema, utilizou-se um Arduino ${ }^{\circledR}$ uno [19] em conjunto com boias de nível, tanto na cisterna inferior, a fim de garantir que não falte água na cisterna inferior para darinício na bomba, quanto na cisterna superior, para não permitir que falte agua e para fazer o desligamento quando a cisterna estiver cheia.

\section{IV.6 PROTÓTIPO}

Com o propósito de realizar testes e garantir a funcionalidade de todo o sistema descrito, desenvolveu-se um modelo em menor escala, utilizando-se de elementos cas eiros. O dimensionamento dos elementos foi realizado conforme foi descrito na seleção de disjuntor da bomba [21]. Obtemos então:

Disjuntor: conforme a equação (5), temos:

$I=\frac{34}{12.7}=0,25 A$, com acréscimo de $20 \%$ de segurança, temos $: I=$ $0,55 A$

Selecionou-se o uso de um disjuntor Siemens Monopolar 5sx1 C1 de 1A. O disjuntor utilizado tem uma margem de tenção de atuação de até $380 \mathrm{~V}$ e $3 \mathrm{kA}$, sendo possível ser utilizado para o experimento [21].

Os medidores de nível foram construídos apenas utilizando apenas a ponta dos fios em contato com a água, sem a estrutura de PVC, pois devido as proporções em menor escala se tornou inviável a elaboração da mesma [22]. Para garantir a segurança, cobrimos os fios com uma camada de termo retrátil [23].

A ligação elétrica também obedece ao que foi descrito anteriormente no trabalho, incluindo apenas um interruptor e duas lâmpadas de LED. A inclusão destes é feita na entrada, antecedendo do disjuntor da bomba, sendo adicionado mais um disjuntor para segurança. Este disjuntor incorpora a ligação comas fontes do Arduino ${ }^{\circledR}$ e com a fonte do monitor de nível, de forma a integrar todos estes elementos em uma única ligação elétrica. A seleção des te é semelhante a seleção do disjuntor para a bomba, por isso se utilizou de um disjuntor de $1 \mathrm{~A}$. É feita a ligação entre o disjuntor da bomba hidráulica, o disjuntor geral e o interruptor, e todo o sistema é alimentado pela corrente elétrica de $110 \mathrm{~V}$. Temos também a inclusão de dois LEDs, um verde e um vermelho que indicam se o sistema está em funcionamento ou não. Estes estão ligados em conjunto com a bomba hidráulica, de forma que quando a bomba estiver ligada, o LED verde acende e quando estiver desligada, o LED vermelho é aceso.

A ligação hidráulica possui uma garrafa PET 2L para representar a cisterna inferior e um suporte de geladeira que representa a cisterna superior. Foi incluído um registro que interliga a cisterna superior e inferior, formando um sistema fechado, o qual utilizou-se para testar todo o sistema de monitoramento e automatização, simulando a falta de água e a confiabilidade do sistema como um todo [24-32].

\section{CONCLUSÃO}

O trabalho foi desenvolvido com o objetivo da elaboração de um sistema de armazenamento de água para residências que sofrem com o problema de abastecimento de água. Muitos bairros da cidade de Manaus sofrem com falta de água, ocasionado pela ineficiência no sistema da concessionária de água em prover o abastecimento regular para toda a população, causando assim dificuldades para as famílias em suas atividades diárias. Em virtude destes problemas, buscamos com este trabalho a elaboração de um sistema autônomo de armazenamento e alimentação de água para uma residência, de forma a auxiliar os moradores em relação aos problemas de abastecimento. Durante a elaboração deste realizamos dimensionamentos residenciais que utilizamos como objeto de estudo, pesquisamos quais os elementos estavam estalados e adicionamos ao projeto os elementos seguros para a utilização. Desenvolveu-se um protótipo onde testamos o modelo de automatização que propomos, para por fim, ser aplicado futuramente na residência.

Foi constatado que a bomba hidráulica dis ponível pode ser utilizada no projeto final, pois a mesma apresenta uma potência compatível para a altura de 4 metros da cisterna já instalada. Entretanto, durante os cálculos verificou-se a necessidade de alteração do encanamento, pois o mesmo possui um diâmetro inferior ao recomendado no catálogo da bomba hidráulica. Em relação a cisterna utilizada para o armazenamento da água obtida do sistema publica, sugere-se a utilização de uma cisterna com o dobro de volume da cisterna que supre a residência, pois se faz necessário sempre que a cisterna de armazenamento tenha água para a alimentação da cisterna que alimenta a residência. Nos testes realizados no protótipo, obtivemos resultados positivos, tanto no funcionamento do acionamento via Arduino ${ }^{\circledR}$, quanto nos sensores de nível. Foi obtido êxito em criar um programa simples e funcional e que dispensasse a atuação do usuário, garantindo segurança e praticidade para o cliente.

Dessa forma, este projeto além da automatização do sistema, da ênfase no monitoramento da operação de abastecimento, levando em conta o quão é importante o acompanhamento da operação, para que seja identificado e diagnosticado com antecedência algum obstáculo ou defeito como sistema antes que comprometa o abastecimento na casa. Levando assim eficiência no abastecimento e diminuindo bastante a porcentagem de ocorrer algumproblema e garantir o abastecimento continuo da água na residência [33-36].

Tendo esse trabalho como objetivo propor um modelo de automatização de um sistema de abastecimento, foramencontradas diversas soluções disponíveis e incorporou-se ao projeto de automatização de um sistema de abastecimento residencial. Foram realizadas medições na residência, dimensionou-se os elementos hidráulicos e elétricos. A bomba hidráulica já instalada é compatível com a altura de $4 \mathrm{~m}$ da cisterna superior. Entretanto foi constatada que a tubulação da residência necessita ser trocada por uma tubulação de 2 polegadas devido a potência que opera a bomba. Foi desenvolvido um sistema autônomo e implantado em um protótipo para testes, e utilizou-se de um Arduino® e um relé de $5 \mathrm{~V}$, para monitoramento desse sistema provou-se funcional e permitiu o acompanhamento do processo de abastecimento residencial em questão. Todo os sensores de nível de monitoramento e de atuação implantados no protótipo foram simulados com sucesso, observando o sistema em menor escala, e obteve-se melhores resultados antes de aplicar no sistema de abastecimento da residência em estudo.

Levando-se em consideração estes aspectos, neste trabalho propomos um modelo funcional de um sistema de abastecimento hidráulico que traz facilidade para pessoas que sofrem como problema de abastecimento em suas residências. Foi possível a elaboração de um projeto simples, que fosse eficiente e que se utilizasse dos elementos já existentes na residência com o 
propósito de reduzir os custos para o cliente final, mas sem descartar a segurança. Possibilitou-se também garantir a praticidade, pois em virtude das dificuldades enfrentadas em garantir a alimentação continua de água para a residência por meio do sistema público, este projeto se utiliza de um sistema autônomo funcional.

Dado os custos para a implantação do sistema propostos sendo alto, sendo necessários diversos itens adicionais que não se encontram em qualquer residência, sendo os itens mais caros as cisternas, devemos destacar que na residência que utilizamos como material de estudo já possuía uma bomba hidráulica e uma cisterna já instalada, o que proporcionou uma economia para o projeto como um todo. Em contrapartida, observa-se que o projeto descrito proporciona uma grande praticidade para o usuário, pois não se faz necessário a atuação. Destaca-se tambémque apenas o sensorque monitora o nível da água e o micro controlador funcionam em tempo integral, garantindo uma maior economia de energia, pois a atuação da bomba só é ativada quando necessário. O trabalho proposto pode ser implementado com novas tecnologias e funcionalidades, visando o controle do consumo de água e a economia financeira, pois estes pontos são sempre relevantes para o usuário final. Entre alguns estudos que podem ser realizados em conjunto com este trabalho destacamos: a implementação de uma unidade de Ethernet no Arduino ${ }^{\circledR}$ para monitoramento a distância; A integração com um aplicativo de celular que realize a medição do custo do consumo de água e o custo de energia com a bomba hidráulica; E um estudo da economia para o usuário final em utilizar o sistema proposto em sua residência, são alguns itens relevantes a serem pesquisados em conjunto com o sistema proposto.

\section{AGRADECIMENTOS}

A JL Automação pelo apoio dado a elaboração do protótipo que utilizamos neste trabalho.

\section{REFERÊNCIAS}

[1] Grassi, Marco Tadeu. As Águas do Planeta Terra, Cadernos Temáticos de Química Nova na Escola. 2001. 40 p.

[2] EBC Agência Brasil. UNESCO: Mundo Precisará Mudar Consumo para Garantir Abastecimento de Água. Mariana Tokarnia. 2015. Disponível em: <agenciabrasil.ebc.com.br/internacional/noticia/2015-03/mundoprecis ara-mudar-consumo-para-garantir-abastecimento-de>. Acesso em 08 de Nov. 2016.

[3] Rebouças, Aldo. Águas Doces no Brasil. São Paulo. 2002.

[4] FUNDAÇÃO NACIONAL DE SAÚDE. Manual de Saneamento. $3^{\circ}$ ed. rev. Brasília. 2006. 408p.

[5] FRANKLIN ELECTRIC INDÚSTRIA DE MOTOBOMBAS. S.A. Manual de Instruções das Bombas e Motobombas. Joinville. $60 \mathrm{p}$.

[5] Carvalho, Roberto de Júnior. Instalações Hidráulicas e o Projeto de Arquitetura. 1 ed. rev. São Paulo. 2013. 89 p.
[6] Brasil Alex N. Máquinas Termohidráulicas de Fluxo. 2010.30p.

[7]iCOS. Automação e Proteção de Bombas Centrífugas. 2016. Dis ponível em: <www.icos.com.br/folhetos-tecnicos/protecao-debomba-cetrifuga/> Acesso em 01 de Nov. 2016

[8] SCHNEIDER MOTOBOMBAS. Termos Hidráulicos Mais Usados em Bombeamento. 2 p. (2016).

[8] Homero, Soares. Máquinas Hidráulicas. Cap. IV. 2012.31p.

[9] Maia, Marcelo. Indicadores de nível de água: Projeto de Montagem que Acompanha o Indicador. 2015. Disponível em: < http://www.inac9v.com.br/2014/02/ao-apertar-o-botao-o-ledindicador.html > Acesso em 09 de nov. 2016 as 12:55pm

[10] Hermini, Helder Aníbal. Sensores de Nível e Capacidade.34p. 2011.

[11] Tsutiya, Milton Tomoyuki. Abastecimento de Água. $3^{\circ}$ ed. São Paulo. 2006. 643 p.

[12] Lakatos, Eva Maria; Marconi, Maria de Andrade. Fundamentos de Metodologia Cientifica. Atlas, 2008.

[13] Gil, Antônio Carlos. Como Elaborar Projetos de Pesquisa. São Paulo. Atlas, 2002.

[14] Massno, Renato. Sistemas de água fria: Dimensionamento das instalações.

http://www.renatomassano.com.br/dicas/residencial/dimensionam ento das instalacoes.asp >. Acesso em 18 de Nov. 2016 as 08:40am.

[15] Figueiredo, Chenia Rocha. Equipamentos Hidráulicos e Sanitários. Brasília. 2007. 91p.

[16] Goodsky. GS Electromagnetc Relay. Taiwan, 2016. 217 p

[17] Grahl, Márcio. Som \& Carro. São Paulo em abril de 1998.n 19.

[18] ABNT - NBR 5626. Instalações Prediais de Água Fria. 1998.

[19] ARDUINO - ARDUINO BOARD UNO: Technical Specs. Disponível em < https://www.arduino.cc/en/Main/ArduinoBoardUno $>$ Acesso em 09 de nov. 2016, as 12:45 pm.

[20] Rebouças, Aldo de C. Água no Brasil: abundância, desperdício e escassez. 2003. 341-345 p.

[21] SIEMENS. Catalogo Disjuntores 5SX1, 5SP, 5SY. São Paulo, 2016. 2p.

[22] Botelho, Manoel. Instalações Hidráulicas

Prediais, Utilizando tubos plásticos. 4 ed. Blucher. 2014. 407p.

[23] Gaya. Catálogo LED 2016. São Paulo. 2016. 40p. 
[24] Mattede, Henrique Mundo da Elétrica: Controle de Nível de Reservatório. 2016. Disponível em: <https://www.mundodaeletrica.com.br/controle-de-nivel-dereservatorio/>Acesso em 10 de Out. 2016.

[25] Mattede, Henrique Mundo da Elétrica: Automação de Reservatório com Arduino e Sensores de Nível.

$2016 . \quad$ Disponível em: <https://www.mundodaeletrica.com.br/automacao-dereservatorio-com-arduino-e-sensores-de-nivel/> Acesso em $10 \mathrm{de}$ Out. 2016.

[26] Brugnari, Arthur; Maestrelli Luiz Henrique. Automação Residencial Via WEB. Curitiba. 2010. 36 p.

[27] Carolino, Ricardo Duarte. Análise do Sistema Hidráulico em Residências. Porto Velho. 2014. 65 p.

[28] Deleski Geovane Assis. Automação de Sistemas de Abastecimento de Água. Porto Velho. 2012. 100p.

[29] PROJETO. Brasil das Águas, Revelando o Azul do Verde e Amarelo. 2016. Disponível em: <brasildasaguas.com.br/educacional/a-importancia-da-agua/> Acesso em 09 de Nov. 2016.

[30] Fortlev. Guia de Instalação: Soluções para Cuidar da Água, Caixa d'água de Polietileno. São Paulo 2015, 5 p.

[31] INSTRUCTABLES. Water Level Indicator with Alarm. Raikut, 2016. Disponível em: < http://www.instructables.com/id/Water-Level-Indicator-with-

Alarm/ > Acesso em 09 de nov. 2016, as 12:50p.

[32] Siqueira, Leila Aparecida. Água Fonte de Vida. Medianeira. 2011. 54 p.

[33] Medeiros, Carlos Fernandes. Abastecimento de Água. Campina Grande. 2006. 147 p.

[34] SO BIOLOGIA. A Água. 2016. Disponível em: <www.sobiologia.com.br/conteudo/Agua/> Acesso em 01 de Out. 2016.

[35] Vital, A. Água no Brasil. 2012. Disponível em: <https://www.portalvital.com/saude/saude/a-agua-no-brasil>

Acesso em 05 de Nov. 2016.

[36] WORLD RESOURCES INSTITUTE. Roots of Resilience Growing the Wealth of the Poor. Washington. 2008. 262 p. 